\title{
Surface Clutter Suppression for Ice Sounding Radars by Coherent Combination of Repeat-Pass Data
}

\author{
Rolf Scheiber, Pau Prats \\ German Aerospace Center (DLR) \\ Microwaves and Radar Institute \\ 82234 Oberpfaffenhofen, Germany \\ Email: rolf.scheiber@dlr.de; pau.prats@dlr.de
}

\begin{abstract}
This paper formulates a new approach for ambiguous surface clutter suppression for ice sounding radars. While surface clutter from the non-orthogonal direction is reduced by means of Doppler and/or SAR processing, the residual surface contributions from across-track may still mask the reflection of internal layers and the bedrock, both coming from the nadir direction. The suggested approach involves several repeated acquisitions separated by certain baselines. It uses a geometry model to derive a synthesized sparse array diagram with adaptive nulls in the direction of the ambiguities. Initial performance evaluations for a P-band space-borne mission indicates that clutter can be suppressed by 30-40 dB. The approach can potentially be demonstrated with airborne sounder data in case precise navigation and motion compensation is ensured.
\end{abstract}

\section{INTRODUCTION}

During the last 2 decades polar research could benefit from a series of radar sounding experiments conducted from ground or with aircrafts, mainly performed on Antarctica [2] and Greenland [3]. Ground measurements are restricted to very small areas and their potential to deliver representative data is limited, although they offer the possibility for controlled experiments providing reference data without being affected by nuisance effects, proper to the airborne or space-borne geometry [4]. The main problem to be considered in the airborne or space-borne case is the presence of clutter (offnadir signals) superimposing the signal of interest, i.e. the reflection of the radar wave from different layers within the ice and from the bedrock. Typical airborne experiments use frequencies in the order of $150 \mathrm{MHz}$ and a low flight altitude, in this way mitigating the problem. In addition Doppler filtering and more recently also focused SAR and Dopplerdelay processing were used to improve the Signal-to-Clutter Ratio (SCR) in along track direction [3]. However, for higher altitude platforms, and especially in the space-borne case, cross-track clutter can completely mask out the signal of interest coming from the nadir direction. For the sounding of Antarctica, a P-band radar is considered for realisation by several Space Agencies, and in this frequency range the ice surface cannot be considered smooth, leading to even higher ambiguous returns than experienced for radars operated at few tens to $200 \mathrm{MHz}$. To cope with this problem, multiaperture or multi-antenna radars were designed (e.g. MARSIS uses a dipole and a monopole [5]) or are in consideration for future missions. However, this solution is accompanied by an increased payload effort. Especially the two aperture case poses strong requirements on the antennas pointing knowledge and symmetry.

Recently, an off-nadir interferometric ice sounding concept has been proposed that enables cross-track clutter suppression by interferometric filtering/removal of the surface signal components [6]. A major advantage of this approach is that a complete swath of several tens of kilometers can be imaged from space (opposite to just a profile as in traditional nadir sounders). Limitations of this concept include less sensitivity to intermediate ice layers due to the side-looking geometry and reduced potential for discrimination/detection of shallow ice depth of few hundreds of meters.

To overcome these limitations and also as a stand-alone alternative nadir-looking concept, a new coherent cross-track clutter suppression method is proposed in this paper. It makes use of coherent combinations of repeated acquisitions of the same or similar platforms. By proper coherent combination of the individual signals and by taking into account the geometric sounder model an adaptive suppression of the surface clutter contribution is achieved. The paper first presents the geometry model and the adaptive algorithm followed by simulations and numerical performance prediction for a spaceborne Pband sounder for Antarctica. The potential demonstration with airborne sounder data is also discussed.

\section{Method For Coherent Clutter Suppression}

Fig. 1 shows the geometry of a nadir looking radar sounder. The flight direction of the airborne or satellite sensor is into the paper plane. The cross-track surface clutter is superimposing the signal of interest from the nadir direction. Note that due to the terrain topography the geometry is generally not symmetrical. Due to physical constraints the antenna size will not realistically be sufficiently large to illuminate only the nadir region of interest. Instead, signals reflected from the left and right of the nadir track are also within the main lobe of the antenna beam. From the knowledge of the geometry, one can compute the directions of surface ambiguity $\theta_{l, r}$ as a function of ice thickness:

$$
\theta_{l, r}=\arccos \frac{H-d h_{l, r}}{H+n \cdot z}=\arccos \frac{H-d h_{l, r}}{R},
$$

where $H$ is the height of the antenna above the reference plane, $d h_{l}$ and $d h_{r}$ are the elevations of the left and right 


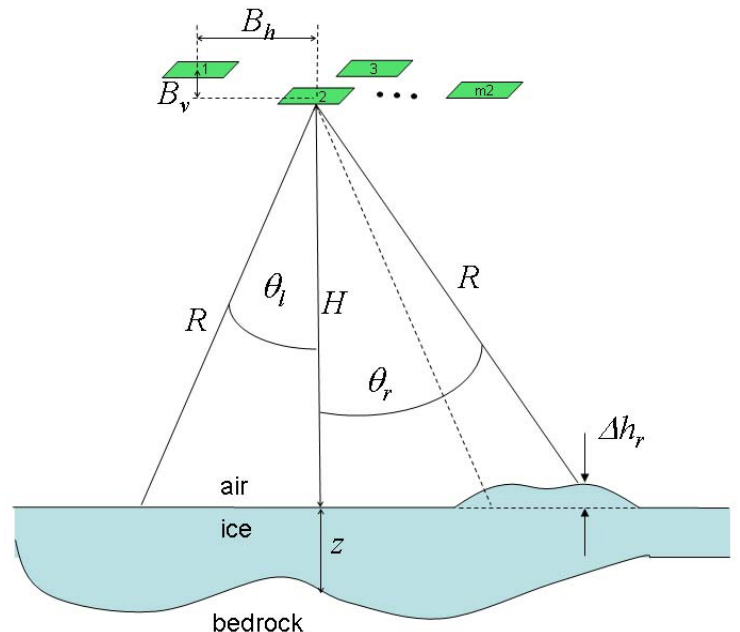

Fig. 1. Cross track sounder geometry with repeated acquisitions

ambiguities above the reference plane, $n$ is the refractive index of ice and $z$ the ice depth from which information is required. The propagation delay measured by the radar is the same for the nadir and off-nadir returns, thus $H+n \cdot z=R$.

Note that in the case of flat surfaces there is a relaxed requirement for the availability of terrain information as $H$ can be estimated from the first strong reflection of the sounder signal and $\theta_{l}=\theta_{r}$.

In a next step the antenna diagram matrix $\mathbf{A}$ is computed and a system of linear equations is set up:

$$
\mathbf{A}_{m_{1} \cdot m_{2}} \cdot \mathbf{w}_{m_{2} \cdot 1}=\mathbf{r}_{m_{1} \cdot 1} .
$$

A is of size $m_{1}$ by $m_{2} . m_{1}$ stands for the number of angles of interest (e.g. 3 corresponding to nadir, and to the first left and first right side ambiguity) and $m_{2}$ for the number of antennas/acquisitions. A contains the complex valued elements of the antenna patterns for the $m_{1}$ directions of interest (e.g. $\theta=0, \theta=\theta_{r}, \theta=\theta_{l}$ ). In case the same antenna is used for all acquisitions, the columns of $\mathbf{A}$ are identical except for an additional phase factor, which is determined by the separation (horizontal and vertical baselines $B_{h}$ and $B_{v}$ ) between the antennas/acquisitions (similar to phased array antennas). Using the parallel ray approximation, the elements of the matrix $\mathbf{A}$ are given as:

$$
\begin{aligned}
& a_{k, i}\left(\theta_{i}\right)=G_{k}\left(\theta_{i}\right) . \\
& \quad \exp \left[\frac{j 2 \pi}{\lambda} \cdot\left(B_{h, k} \sin \theta_{i}+B_{v, k} \cos \theta_{i}\right)-\phi_{c a l, k}\right],
\end{aligned}
$$

where $G_{k}$ is the complex valued gain for antenna/acquisition $k$, and $\theta_{i}$ is the angle of interest. The calibration phase $\phi_{c a l, k}$ accounts for different propagation phase offsets (e.g. due to atmospheric and ionospheric effects) and may be estimated independently for each data acquisition $k$ from the phase of the reflection of the air ice interface (i.e. the first strong echo).

The column vector $\mathbf{r}$ in eq. 2 contains the information about the resulting gain of the constellation. For instance

$$
\mathbf{r}=\left[\begin{array}{l}
c \\
0 \\
0
\end{array}\right], c \neq 0,
$$

for the three angles $\theta=0, \theta=\theta_{r}$, and $\theta=\theta_{l}$. This ensures signal cancellation in the directions of the surface clutter $\theta_{r}$ and $\theta_{l}$ and no cancellation in the nadir direction. The linear system in eq. 2 can be solved in case there is a right inverse for the matrix A [7]. For the application under consideration this is usually the case (number of acquisitions $m_{2}$ larger than the number of angles of interest $m_{1}$ ) and the complex weights $\mathbf{w}$ for the different acquisitions can be obtained as:

$$
\mathbf{w}_{m_{2} \cdot 1}=\mathbf{A}_{m_{2} \cdot m_{1}}^{T} \cdot\left[\mathbf{A} \cdot \mathbf{A}^{T}\right]_{m_{1} \cdot m_{1}}^{-1} \cdot \mathbf{r}_{m_{1} \cdot 1} .
$$

The final step is the weighted addition of the individual acquisitions to generate a diagram with surface clutter cancelled to best possible extend (nulled). The undistorted radar reflectivity of nadir signals is then given as:

$$
\sigma(z)=\frac{S_{\text {sum }}(z)}{\sum_{k=1}^{m_{2}} w_{k}(z) G_{k}(0)} \cdot L^{-1}
$$

where $S_{\text {sum }}$ is the weighted sum of all acquisitions and $L$ is the loss due to propagation into the ice medium, which depends on ice properties and temperature. A figure of merit is given by the integrated power ratio of the synthesised pattern in the vicinity of nadir and ambiguous clutter directions (integrated signal to clutter ratio $I S C R$ ).

$$
I S C R=\frac{\int_{\theta=-\Delta \theta_{F}}^{\Delta \theta_{F}} G_{\text {sum }}(\theta) d \theta}{\int_{\theta=\theta_{l}-\Delta \theta_{C}}^{\theta_{l}+\Delta \theta_{C}} G_{\text {sum }}(\theta) d \theta+\int_{\theta=\theta_{r}-\Delta \theta_{C}}^{\theta_{r}+\Delta \theta_{C}} G_{\text {sum }}(\theta) d \theta} .
$$

The following choice of $\Delta \theta_{F}$ and $\Delta \theta_{C}$ is suggested. The integration border $\Delta \theta_{F}$ is given by the nadir Fresnel reflection footprint:

$$
\Delta \theta_{F}=\tan ^{-1}\left(\frac{\sqrt{H \lambda / 2}}{H}\right)
$$

whereas $\Delta \theta_{C}$ is determined by the range resolution projected onto the ground:

$$
\Delta \theta_{F}=\cos ^{-1}\left(\frac{H}{R+\rho_{r}}\right)-\cos ^{-1}\left(\frac{H}{R}\right)
$$

The figure of merit in eq. 7 can also be used to quantify the effect of different error sources (e.g. inexact baseline length, unknown topography or slope).

\section{Spaceborne P-BAnd SOUnder Simulation}

The suggested clutter suppression method is evaluated for a space-borne geometry for the sounding of Antarctica in P-band. Table I summarizes the parameters. Fig. 2 and 3 show the gain of one single antenna (black) and for the sparse array (green) synthesized from 3 evenly and from 7 unevenly sampled acquisitions. The results are depicted for 

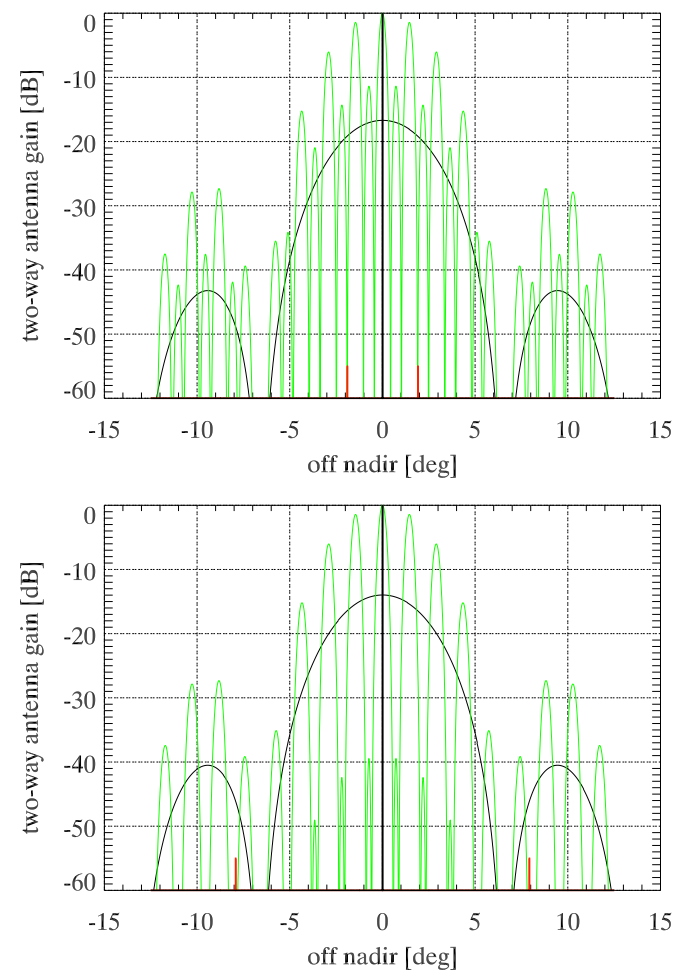

Fig. 2. Sparse array gain for 3 acquisition scenario and 200m (upper plot) and $3000 \mathrm{~m}$ (lower plot) ice depth. Orbit separation is $[-27,0,27] \mathrm{m}$.
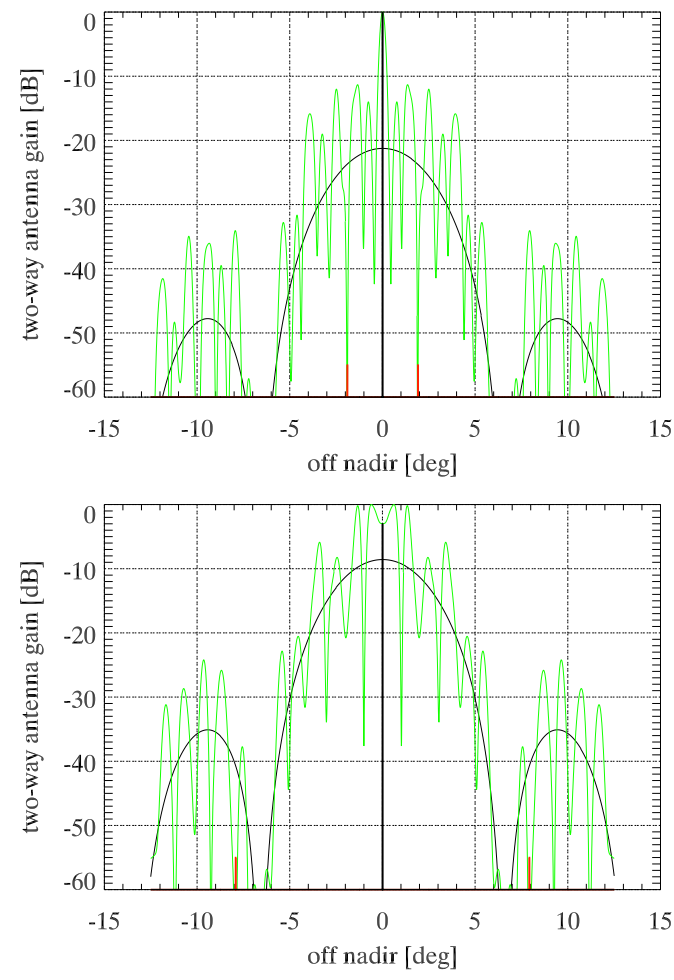

Fig. 3. Sparse array gain for 7 acquisition scenario and $200 \mathrm{~m}$ ice depth. Orbit separation is $[-50,-20,-13,0,10,26,45] \mathrm{m}$.
TABLE I

SiMULATION PARAMETERS FOR P-BAND SPACEBORNE CASE

\begin{tabular}{|c||c|}
\hline Parameter & Value \\
\hline \hline carrier frequency [MHz] & 435 \\
\hline platform altitude $[\mathrm{km}]$ & 650 \\
\hline antenna length $[\mathrm{m}]$ & 6 \\
\hline range bandwidth $[\mathrm{MHz}]$ & 6 \\
\hline no of acquisitions & $3 \& 7$ \\
\hline orbit separation $[\mathrm{m}]$ & $10-30$ \\
\hline \hline
\end{tabular}

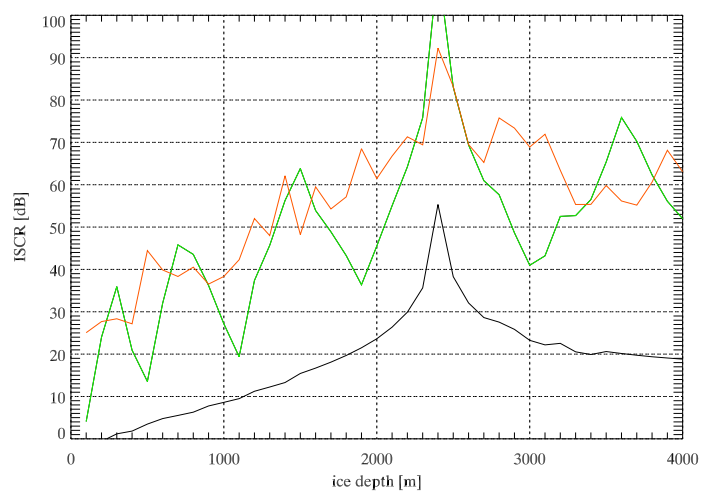

Fig. 4. ISCR for single antenna (black), sparse array constellation of three (green) and seven (red) acquisitions.

two different ice depths $(200 \mathrm{~m}$ and $3500 \mathrm{~m})$. The location of the left and right ambiguity angles according to eq. 1 is marked with red. Note the adaptive steering of zeroes in the direction of the clutter ambiguity angles, whereas increased gain is present in the nadir direction. The across-track ambiguities for ice depth exceeding $2400 \mathrm{~m}$ (corresponding to $6.5 \mathrm{deg}$ off-nadir) are covered by the sidelobes of the antenna for this selected geometry and antenna aperture size. Of course this has influence on the performance.

The investigation of the $I S C R$ according to eq. 7 is plotted in Fig. 4. The black line indicates the clutter suppression performance for a single aquisition/antenna. As no height variation (zero cross track slope) is assumed, there is a maximum of the ISLR at the location of the null of the antenna pattern at $2400 \mathrm{~m}$ ice depth. For the 3 aquisition scenario (green line) there are regions with poor ISCR corresponding to low gain of the synthetized sparse array pattern (in this case at ice depth around 500, 1100, 2900, and 3000m). However, for considerable regions of ice depths the clutter suppresion performance could be improved by more than $30 \mathrm{~dB}$. The improvement when 7 tracks are available is twofold (red line). The average clutter suppression is improved (up to $40 \mathrm{~dB}$ ) and there are no invalid depths.

In order to select valid regions one needs to consider relative estimates of nadir reflectivity (from internal layers and bedrock) and surface clutter. These estimates are used to find the areas where the ISCR is sufficiently high to allow the detection of the features of interest. For example in the 3 acquisition scenario, some areas need to be excluded whereas 
TABLE II

ICE PROFILE SIMULATION PARAMETERS

\begin{tabular}{|c||c|}
\hline \multicolumn{1}{|c||}{ Parameter } & Value \\
\hline \hline attenuation $[\mathrm{dB} / \mathrm{km}]$ & 10 \\
\hline internal layer depth $[\mathrm{m}]$ & $671,1118,1565$ \\
\hline internal layer refl $[\mathrm{dB}]$ & -10 \\
\hline bedrock depth $[\mathrm{m}]$ & 2236 \\
\hline bedrock reflectivity $[\mathrm{dB}]$ & 0 \\
\hline SNR of bedrock reflection $[\mathrm{dB}]$ & 10 \\
\hline \hline
\end{tabular}

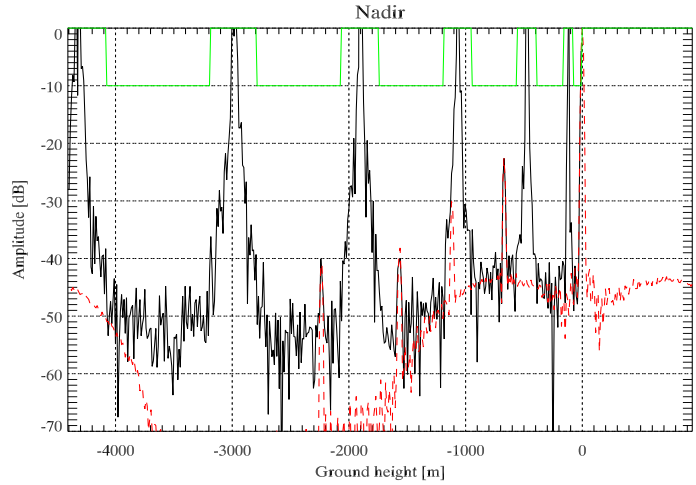

Nadir

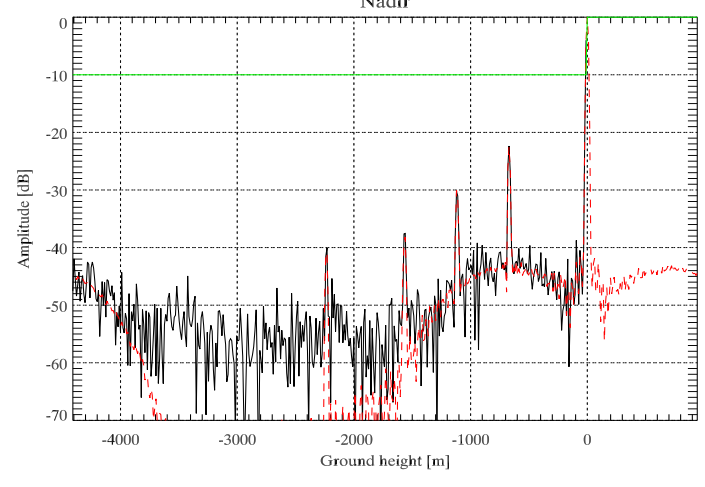

Fig. 5. Ice depth profile reconstruction with sparse array constellation of three (up) and seven (bottom) acquisitions. Reference nadir signal (red), reconstruction (black), non-valid areas (green at $0 \mathrm{~dB}$ ).

in case 7 tracks are available the complete ice profile can be reconstructed at sufficient clutter suppression performance.

A complete reconstruction of a depth profile is shown in Fig. 5 for the 3 and 7 acquisitions scenario, respectively. Simulation parameters are provided in Table II. Red is the simulated clutter-free signal and black is the reconstructed signal. Invalid areas in the three acquisition scenario are indicated by the green line at the $0 \mathrm{~dB}$ level. They were evaluated as a function of the sparse array relative to the single antenna gain. These regions correspond to the areas of low ISCR in Fig. 4.

The investigation of different error sources (i.e. required orbit knowledge accuracy and ice surface topography) is an ongoing task.

\section{Airborne Sounder: Experimental Aspects}

The coherent clutter suppression method presented in section II can potentially be demonstrated with airborne sounder data. However, for the airborne case, several additional aspects must be considered:

- The demand for parallel tracks poses strong requirements on the accuracy of aircraft navigation.

- Due to the low altitude of operation the desired distance between the tracks is in the order of a few meters, only.

- Processing generally needs to involve motion compensation for focussing the along-track dimension. This inherently comes with a conflict as different motion compensation is to be applied for nadir, left and right ambiguity.

- residual motion errors may impact the reconstruction accuracy.

Nevertheless, a limited number of sounder tracks have been acquired by the E-SAR system at $350 \mathrm{MHz}$ in the frame of the ICESAR campaign performed in spring 2007 in Svalbard. These data are presently under evaluation.

\section{CONCLUSION}

The coherent combination of spatially separated repeated acquisitions as presented in section II can equally be appplied to multiple aperture antennas operated from one single satellite. In this case at least three simultaneous receive channels must be available on-board. Besides demonstration with airborne data further simulations are required to consolidate the reconstruction accuracy for non-ideal scenarios. This will need to take into consideration temporal instabilities due to glacier flow, atmosphere and ionosphere. For an extraterrestrial application the orbit restituation accuracy might be a limiting factor.

\section{ACKNOWLEDGMENT}

This work is performed within the ACRAS study, ESA contract no 19961/06/NL/HE. The authors would like to thank ESA and the complete ACRAS team for the discussions on the presented topic.

\section{REFERENCES}

[1] J.D. Paden, C.T. Allen, S. Gogineni, K.C. Jezek, D. Dahl-Jensen, L.B. Larsen: Wideband Measurements of Ice Sheet Attenuation and Basal Scattering, IEEE Geosc. Rem. Sens. Lett., vol. 2, no. 2, pp. 164-168, April, 2004.

[2] J.W. Holt, M.E. Peters, S.D. Kempf, D.L. Morse, D.D. Blankenship : Echo source discrimination in single-pass airborne radar sounding data from the Dry Valleys, Antarctica: Implications for orbital sounding of Mars, J. Geophys. Res., vol. 111, July, 2006.

[3] D.A. Braaten, S. Gogineni, D. Tammana, S. Namburi, J. Paden, K.K. Gurumoorthy : Improvement of Radar ice-thickness measurements of Greenland outlet glaciers using SAR processing, Annals of Glaciology, vol. 35, pp.73-78, 2002.

[4] J.D. Paden, C.T. Allen, S. Gogineni, K.C. Jezek, D. Dahl-Jensen, L.B. Larsen: Wideband Measurements of Ice Sheet Attenuation and Basal Scattering, IEEE Geosc. Rem. Sens. Lett., vol. 2, no. 2, pp. 164-168, April, 2004.

[5] G. Picardi, S. Sorge, R. Seu, G. Fedele, R.L: Jordan, Coherent Cancellation of Surface Clutter for Radar Sounding, IGARSS Proceedings, Hamburg, Germany, 1999.

[6] E. Rodriguez, E., A. Freeman, K. Jezek, X. Wu, A New Technique for Interferometric Sounding of Ice Sheets EUSAR Proceedings, Dresden, Germany, 2006

[7] G. Strang: Linear Algebra and its Applications,ISBN: 0155510053, 1988. 